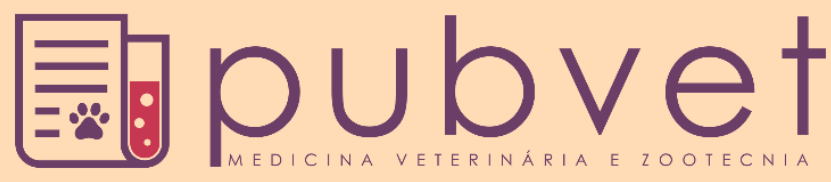

https://doi.org/10.31533/pubvet.v16n01a1002.1-8

\title{
Indigestão vagal em bovinos
}

\author{
Ana Paula Amorim da Costa ${ }^{1}$, Gabriela Gomes Carvalho da Silva ${ }^{2}{ }^{\bullet}$, Cristiane Lopes \\ Mazzinghy $^{3^{*} \bullet}$, Guilherme Augusto Motta ${ }^{\circ}$, Erycka Carolina França ${ }^{5}$, Fernanda Luz Alves \\ Neves $^{30}$, Josemara Silva Santos ${ }^{60}$, Mildre Loraine Pinto ${ }^{60}$ \\ ${ }^{I}$ Médica Veterinária, residente multiprofissional em saúde coletiva pela Fundação Escola de Saúde Pública; Palmas-TO, Brasil \\ ${ }^{2}$ Graduanda em medicina veterinária pela Faculdade de Ciências do Tocantins, Araguaína-TO, Brasil. \\ ${ }^{3}$ Professora do curso de medicina veterinária da Faculdade de Ciências do Tocantins, Departamento de medicina veterinária, Araguaína-TO, Brasil. \\ ${ }^{4}$ Professor do curso de medicina veterinária da Universidade de Araraquara, Departamento de medicina veterinária, Araraquara-SP, Brasil. \\ ${ }^{5}$ Médica veterinária autônoma, Marabá-PA, Brasil. \\ ${ }^{6}$ Professora do curso de medicina veterinária da Centro Universitário Luterano de Palmas, Departamento de medicina veterinária, Palmas-TO, Brasil. \\ *Autor para correspondência, E-mail: crislp03@yahoo.com.br
}

Resumo. A indigestão vagal é definida como uma patologia gastrointestinal não infecciosas dos ruminantes, caracterizada por distúrbios que acometem o nervo vago, e causam alterações motoras nos pré-estômagos desses animais. A indigestão vagal se baseia em quatros tipos: O tipo I acontece pela falha do esvaziamento ruminal por disfunção cárdica, o tipo II por uma estenose funcional anterior com hipermobilidade, o tipo III ocorre em função da estenose funcional posterior, enquanto que o tipo IV é identificada como indigestão de gestação tardia. Para o diagnóstico necessita-se de exames clínicos associado a laboratoriais por se tratar de sinais clínicos inespecíficos que variam de acordo com o local de comprometimento do nervo vago. $\mathrm{O}$ paciente diagnosticado com indigestão vagal apresenta prognóstico desfavorável. O tratamento consiste em sessões terapêuticas, administração de bicarbonato, fistulação e/ou canulação do rúmen, e até mesmo, por causa da etiologia multifatorial da indigestão vagal, procedimentos cirúrgicos. Conclui-se, então, que, a indigestão vagal é uma síndrome de grande importância na medicina veterinária, que acomete e causa desordens no trato digestório dos bovinos, e estudar sobre essa enfermidade se faz necessário para garantir e tornar mais amplo o conhecimento de doenças que acometem os ruminantes.

Palavras-chave: Trato digestório, nervus vagus, ruminantes, síndrome

\section{Vagal indigestion in cattle}

Abstract. The vagal indigestion is defined as a non-infectious gastrointestinal pathology
of ruminants, characterized by disorders that affect the vagus nerve, and cause motor
changes in the pre-stomach of these animals. Vagal indigestion is based on four types: type
I is caused by failure of rumen emptying due to cardiac dysfunction, type II is caused by
an anterior functional stenosis with hypermotility, type III is caused by posterior functional
stenosis, while type IV is identified as late gestation indigestion. The diagnosis requires
clinical and laboratory tests, because the clinical signs are non-specific and vary according
to where the vagus nerve is involved. The patient diagnosed with vagal indigestion has an
unfavorable prognosis. Treatment consists of therapy sessions, administration of
bicarbonate, fistulation and/or cannulation of the rumen, and even, because of the
multifactorial etiology of vagal indigestion, surgical procedures. It can be concluded, then,
that vagal indigestion is a syndrome of great importance in veterinary medicine, which
affects and causes disorders in the digestive tract of bovines, and a study on this disease is
necessary to ensure and broaden the knowledge of diseases that affect ruminants

Keywords: Digestive tract, ruminant, syndrome, vagus nerve 


\section{Indigestión vagal en ganado}

Resumen. La indigestión vagal se define como una patología gastrointestinal no infecciosa de los rumiantes, caracterizada por trastornos que afectan al nervio vago, y que provocan cambios motores en el pre-estómago de estos animales. La indigestión vagal se basa en cuatro tipos: el tipo I se produce debido al fallo del vaciado del rumen por disfunción cardíaca, el tipo II por una estenosis funcional anterior con hipermotilidad, el tipo III se produce debido a una estenosis funcional posterior, mientras que el tipo IV se identifica como indigestión de gestación tardía. Para el diagnóstico es necesario exámenes clínicos asociados a exámenes laboratoriales ya que se trata de signos clínicos inespecíficos que varían de acuerdo con el lugar afectado del nervio vago. El paciente diagnosticado de indigestión vagal tiene un pronóstico desfavorable. El tratamiento consiste en sesiones terapéuticas, administración de bicarbonato, fistulación y/o canulación del rumen, e incluso, debido a la etiología multifactorial de la indigestión vagal, procedimientos quirúrgicos. Por lo tanto, se concluye que la indigestión vagal es un síndrome de gran importancia en medicina veterinaria, que afecta y provoca trastornos en el tracto digestivo de los bovinos, y es necesario estudiar esta enfermedad para garantizar y ampliar el conocimiento de las enfermedades que afectan a los rumiantes.

Palabras clave: Tracto digestivo, nervus vagus, rumiantes, síndrome

\section{Introdução}

As complicações gastrointestinais que atingem os bovinos estão ligadas às questões nutricionais e de manejo e tendo sido constatadas constantemente em casos de atendimento clínico destes animais (Burns et al., 2013). Conhecida também como síndrome de Hoflund, a indigestão vagal é um comprometimento que ocorre por inflamação, comprensão ou lesão do nervo vago (total ou parcial) no decorrer do seu trajeto (Rehage et al., 1992). As estruturas envolvidas na ocorrência desta indigestão são responsáveis pelas funções nervosas e musculares dos pré-estômagos de ruminantes (Smith, 2006).

O nervo vago se fraciona no início do tórax, segue em direção pelo mediastino e se divide sobre o pericárdio em ramos, que, posteriormente, forma o tronco vagal dorsal (supre o fígado e o plexo gástrico) e o tronco vagal ventral que inerva o plexo renal, mesentérico, gonadal e celíaco. Danos no nervo vago e em seus ramos podem surgir de diversas maneiras, podendo acometer o processo de deglutição, o coração, e outras vísceras (Dyce et al., 2004).

Definida como uma alteração na passagem da ingesta, a indigestão vagal pode ser classificada em estenose funcional anterior, quando há distúrbios no orifício retículo-omasal e estenose funcional posterior, quando há modificações no caminho de ingesta pelo piloro (Constable et al., 2016).

Sabendo-se da frequência de indigestão vagal na clínica de ruminantes objetiva-se com esta revisão registrar informações anatômicas do sistema digestório dos bovinos bem como abordar aspectos clínicos e tratamento da enfermidade.

\section{Anatomia e fisiologia do trato gastrointestinal}

O sistema digestório compreende os órgãos desde a boca até o ânus. Os ruminantes possuem boca, constituída de língua e dentes (são heterodontes e difiodontes) porém, em sua arcada dentária superior apresentam pulvino dentário no lugar dos dentes incisivos e caninos. Esta região do sistema digestório é responsável por capturar, triturar e insalivar o alimento que seguirá pela faringe, esôfago até os préestômagos e estômago (Köning \& Liebich, 2011).

Os bovinos são mamíferos herbívoros e possuem o estômago composto por quatro cavidades denominadas: rúmen, retículo, omaso (pré-estômagos) e abomaso (estômago). As câmaras anteriores ao estômago simples são revestidas de epitélio escamoso aglandular, onde o alimento é submetido a digestão microbiana antes de ser sujeito a digestão química no divertículo glandular (Frandson et al., 2011). 
Sobre as características anátomo-fisiológicas, na região dorsal comum ao retículo e ao rúmen está a abertura do esôfago, região do cárdia. A sua mucosa possui duas pregas que formam um tubo fechado e estende-se até o omaso, chamado de sulco ruminorreticular. Este é usado pelos bezerros na fase de mama para o desvio do leite do rúmen e retículo (Reece \& Penteado Júnior, 1996). A mucosa reticular tem cristas que caracterizam seu nome vulgar de "favo de mel". Devido essa característica e ao hábito alimentar não seletivo dos bovinos, os objetos estranhos eventualmente ingeridos tendem a permanecer nesta região e gerar lesões aos serem movimentados com as contrações do órgão, suscitando peritonite traumática ou reticulo-pericardite traumática (Köning \& Liebich, 2011).

O omaso é esférico e fica à direita do rúmen-retículo e caudal ao fígado, é preenchido por lâminas musculares folhosas paralelas e sua mucosa é composta por células estratificadas pavimentosas com papilas curtas. Possui duas fases de contração, a primeira fase pressiona o alimento nos recessos do omaso para reabsorção de água e na segunda parte encaminha os conteúdos desidratados ao abomaso (Frandson et al., 2011).

Após os pró-ventrículos, encontra-se o abomaso, este divertículo é a parte glandular do sistema digestório dos ruminantes. Ele é dividido em fundo gástrico, corpo do estômago e piloro, ambos glandulares. Todavia, seu epitélio apresenta duas regiões glandulares distintas denominadas como região fúndica (fundo) e pilórica. É topograficamente influenciado pela atividade dos pró-ventrículos, mas encontra-se anatomicamente ventral ao omaso e caudal à direita do rúmen. Apresenta curvatura maior (côncava) voltada para o ventre e a curvatura menor (convexa) voltada para o dorso do animal (Köning \& Liebich, 2011).

Estas vísceras abdominais são inervadas pelo nervo vago (nervus vagus). Este se divide em direito e esquerdo no mediastino, atravessa o hiato esofágico e forma um plexo neural ao se espalhar e unir-se as fibras simpáticas na cavidade abdominal formando troncos vagais ventral e dorsal (Köning \& Liebich, 2011). Sua função é regular a motricidade das câmaras digestivas (Radostits et al., 2010). A região de cárdia, o saco ventral do rúmen, o retículo, omaso, abomaso e o piloro são inervados pelo nervo vago ventral. Enquanto que, a inervação vagal dorsal, atinge cárdia, saco dorsal do rúmen, retículo, omaso e abomaso (Feitosa, 2014; Hedlund \& Fossum, 2008; Willard, 2010).

A fisiologia da digestão dos ruminantes começa pela boca com a trituração do alimento em partículas menores, sendo que após o alimento ser apreendido, ele passa pela mastigação sendo insalivado. A saliva possui função de amolecimento e lubrificação da ingesta, facilitando a mastigação e posterior deglutição. Os bovinos podem produzir até 200 litros de saliva por dia, essa grande quantidade permite que o conteúdo ruminal permaneça líquido e não forme espuma (Frandson et al., 2011).

A saliva possui a capacidade de tamponamento, estimulação da fermentação e transporte de fluido e condução da ingesta no retrocesso a boca para a ruminação e ao longo do estômago para o intestino delgado. A capacidade tamponante se deve às altas concentrações de bicarbonato e de fosfato presentes na saliva desses animais (Teixeira, 1996; Welkoborsky, 2011).

O sistema enzimático da digestão dos ruminantes depende de microrganismos que fazem a digestão de fibras vegetais. Estes localizam-se no rúmen (câmara fermentativa), onde os carboidratos estruturais dos vegetais são desdobrados em ácidos graxos voláteis (AGV's) úteis ao metabolismo do animal (Berchielli et al., 2011; Clauss \& Rössner, 2014). Os AGV's produzidos pela ação fermentativa são ácido acético, o ácido propiônico e o ácido butírico que são absorvidos nos pró-ventrículos, sendo eles a principal fonte de energia para os ruminantes. A digestão microbiana também produz gases, metano e dióxido de carbono, eles se acumulam na câmara sob o alimento ingerido (Frandson et al., 2011). Quando a produção de gás é maior do que a eliminada pela eructação, há um aumento do rúmen e do retículo denominado timpanismo. Esse volume aumentado dos pró-ventrículos pressiona a cavidade torácica, causando dificuldade respiratória, alterações cardíacas, inapetência e motilidade do rúmen e retículo anormal (Frandson et al., 2011).

O rúmen e retículo tem uma sequência complexa e frequência variável de contrações que se repetem diversas vezes por minuto ( 2 a 3 movimentos de mescla em 3 minutos) permitindo a mistura do conteúdo alimentar e promovendo tanto a fermentação quanto a passagem para as outras câmaras (Reece, 2008). Desse modo, no ruminante, após a ingestão, o alimento retorna à cavidade oral para ser insalivado e mastigado novamente, referindo-se então o termo ruminante a esse hábito alimentar. Basicamente, o 
alimento é triturado e insalivado na boca e então, enviado ao rúmen onde os microrganismos fazem a digestão fermentativa. As partículas maiores que chegam ao rúmen são enviadas ao retículo e do retículo são direcionadas novamente para a boca para nova mastigação e insalivação, prosseguindo assim, o bolo ao omaso (Reece, 2008). Para chegar ao omaso, o alimento passa pelo orifício retículo-omasal, neste órgão não há uma cadência como é observada no retículo. Neste divertículo também ocorrem fermentação e absorção de água, AGV's e eletrólitos. Apesar de ser o menor compartimento, é o local de maior absorção de nutrientes, além de regular a propulsão do alimento até o abomaso (Reece, 2008). O abomaso é responsável pela digestão enzimática liberando hormônios e suco gástrico. O suco gástrico contém ácido clorídrico, pepsinogênio, muco, enzima renina, água e fator intrínseco. As liberações destas substâncias são reguladas por estímulos sensoriais, pela presença do conteúdo alimentar e pela liberação de alguns hormônios inibidores de ácido clorídrico no duodeno. As contrações do abomaso misturam o alimento, continuam a digestão mecânica e força o alimento a passar o esfíncter pilórico (Reece, 2008).

\section{Indigestão vagal}

Alterações no trato gastrointestinal (TGI) de ruminantes são um empecilho grave na criação. Entre os distúrbios do TGI, observa-se a indigestão vagal ou síndrome de Hoflund, como foi denominada inicialmente (Rehage et al., 1992). Esta doença pertence a um grupo de afecções gastrointestinais não infecciosas dos ruminantes, caracterizada pelo desenvolvimento gradual da distensão abdominal secundária à distensão do rúmen e retículo, sendo dividida em quatro tipos, I, II, III e IV (Foster, 2017).

Hoflund (1940) descreveu quatro tipos de indigestão vagal baseada no suposto local de lesão vagal. O tipo I é caracterizado pela falha do esvaziamento ruminal por disfunção cárdica (estenose funcional anterior com hipomotilidade), o tipo II por uma estenose funcional anterior com hiper motilidade. $\mathrm{O}$ tipo III ocorre em função da estenose funcional posterior, enquanto que o tipo IV é identificada como indigestão tardia da gestação. Os tipos I e IV são considerados raros e a categorização é de baixa relevância clínica. Descoberta em 1940, a partir da simulação de distúrbios no estômago de bovinos após vagotonia seletiva, produzindo indigestão crônica. Essa pesquisa, coordenada pelo pesquisador Hoflund, demonstrou que o principal fator desencadeador da doença era uma lesão no nervo vago (Hoflund, 1940).

A indigestão vagal do tipo I é resultante da distensão ruminal causada pelo gás livre que resultará em um rúmen hipomotílico ou atônico e provocará tensão abdominal aumentada (Perkins, 2017). Ligados a indigestão vagal tipo I e à disfunção ruminal, os traumas de faringe, bloqueios causados por corpos estranhos, compressão esofágica e circunstâncias patológicas presentes no mediastino são causas potenciais para o acontecimento da síndrome caracterizada por falha na eructação e distensão gasosa do rúmen (Dirksen et al., 2005; Smith et al., 1992).

O tipo II desenvolve-se a partir de condições que impeçam a passagem de ingesta pelo canal omasal para o abomasal. As causas mais comuns são os abcessos reticulares e hepáticos que se desenvolvem na parede direita ou medial ao retículo. Pode ocorrer também por obstrução mecânica devido a ingestão de corpos estranhas ou pela presenção de papilomas ou granulomas. A falha no transporte omasal pode ocasionar distensão ruminoreticular crônica (Rehage et al., 1992). Conhecida como estenose funcional posterior ou indigestão vagal do tipo III, esse tipo de síndrome apresenta comprometimento do fluxo da ingesta através do piloro, no qual sucede em acúmulo de alimento na parte interna do abomaso, acometendo o fluxo do bolo alimentar no trecho entre abomaso e intestino (Constable et al., 2016).

A indigestão vagal do tipo IV não é bem definida, ocorre em fêmeas prenhes, mais precisamente no terço final da gestação, sendo conhecida também por indigestão do final da gestação. Acredita-se que esse tipo ocorra devido ao aumento uterino causando deslocamento cranial do abomaso e assim, inibindo o esvaziamento abomasal (Hussain et al., 2014).

\section{Sinais clínicos}

Bovinos com indigestão vagal apresentam alteração evidente da motilidade em algumas formas apresentação da doença. Também em sua fase aguda podem apresentar dispneia acentuada, elevação média dos batimentos cardíacos até 100-120/minuto, sopro transitório durante a sístole, sendo audível e 
notado no timpanismo ruminal causado por indigestão vagal. Além disso, sinais como inapetência, desidratação, ausência ou escassez de fezes, anorexia, cetose, motilidade anormal ou reduzida do rúmen e retículo, sons anormais na percussão e auscultação da zona lateral esquerda abdominal são afecções diferenciais mais comuns na indigestão vagal e outras enfermidades (Radostits et al., 2010). Em alguns casos devido à desidratação é observado bradicardia com progressão para taquicardia no decorrer da progressão da doença (Radostits et al., 2010). Geralmente a temperatura e a frequência respiratória encontram-se na normalidade; porém, em casos de etiologia múltipla esses parâmetros podem aumentar (Romão et al., 2012; Simões et al., 2014).

Na palpação, nota-se o rúmen distendido por espuma ou gás que ao ocupar todo o abdômen empurra o rim esquerdo para a direita da linha média, o saco ventral do rúmen também é palpável na mesma região. Já na metade inferior do lado direito é palpável o abomaso compactado (Radostits et al., 2010).

\section{Diagnóstico}

O diagnóstico é baseado no histórico do animal e da propriedade, anamnese, exame físico e exames complementares, ou seja, é clínico associado ao laboratorial. Esta associação permitirá tanto o diagnóstico da doença, quanto a identificação da causa primária e sua classificação. Em casos de indigestão, o prognóstico costuma ser desfavorável (Radostits et al., 2010).

Pode ser usado como recurso a ultrassonografia, que permitirá visibilizar a função reticular, que apresenta contrações com intervalos regulares e, dependendo da causa, apresentará frequência, amplitude e velocidade alteradas. E pode servir de diagnóstico para reticulopericardite traumática, uma das causas primárias mais comuns; porém, as vezes não é possível a identificação dessa patologia (Foster, 2017).

Embora distúrbios relacionados com a indigestão vagal possam ocorrer em qualquer época do ano, Soares et al. (2021) sugerem que em países tropicais estes são mais prevalentes durante a estação seca, que é o período de escassez de forragem de qualidade.

\section{Tratamento}

Em casos de emergências, as vezes não é possível um exame físico completo, sendo necessário uma intervenção imediata. Inicialmente, deve-se considerar a descompressão gástrica, pois à medida que o rúmen se distende o animal vai perdendo a capacidade de respirar. É recomendado o uso da sonda orogástrica, visto que as descompressões com trocarte podem levar a quadros de peritonite (Foster, 2017). Em casos menos graves, promover a salivação ajuda a desnaturar a espuma presente no rúmen, ou administrar bicarbonato que produzirá também um efeito tampão. $\mathrm{O}$ animal deve ser submetido a uma mudança de alimentação, e estimulando ao processo de eructação (Radostits et al., 2010). Tratamentos mais invasivos podem ser necessários, como a fistulação e/ou canulação do rúmen. São procedimentos cirúrgicos eficazes em casos de timpanismo recidivante (Muzzi et al., 2009). Mas, em alguns casos o tratamento consiste de outras abordagens terapêuticas devido a etiologia multifatorial da indigestão vagal.

\section{Relatos clínicos de indigestão vagal}

Reis et al. (2016) relatam a primeira ocorrência de indigestão vagal em bovinos zebu criados de forma extensiva no Brasil. Os autores registram ocorrência de três casos de indigestão vagal durante um estudo epidemiológico objetivando informações sobre criação, pastagem e manejo sanitário em propriedades do município de Açailândia no Maranhão e em municípios do nordeste do Pará. Foi descrito o caso em uma vaca nelore de quatro anos (caso 1) e em dois bovinos, cruzamento de Nelore e Zebu, de um ano, sendo uma fêmea (caso 2) e um macho (caso 3). Estes eram criados com a espécie de capim Brachiaria brizantha. Os animais foram examinados durante visitas a três propriedades diferentes, e evidenciaram entre os sinais clínicos a falta de apetite, desidratação leve, pelagem áspera e estado nutricional normal. Após o exame clínico do sistema digestivo, foi notado movimentos ondulantes na fossa para lombar esquerda, distensão bilateral do abdome, e as regiões ventral e dorsal estavam distendidas no lado esquerdo, e o flanco ventral direito indicava o abdômen de aspecto maçã- 
pera. Além disso, na ausculta dos pré-estômagos, contrações ruminais incompletas foram observadas com frequência normal no animal 1 e com frequência aumentada nos animais 2 e 3 . O animal 1 apresentava diarreia e presença de fezes nas superfícies das tuberosidades isquiáticas; porém, os animais 2 e 3 apresentavam fezes escassas com uma consistência firme.

Como exame complementar foi requerido o hemograma completo, que não revelou anormalidades em nenhum dos três casos. Porém, ao realizar-se o teste de atropina (realizado de acordo com as técnicas descritas por Dirksen et al. (2005) confirmou-se a suspeita clínica de indigestão vagal em todos os três animais, levando ao aumento da frequência cardíaca em pelo menos $16 \%$ após a aplicação do sulfato de atropina (Reis et al., 2016).

A pesquisa não demonstra intervenção terapêutica de forma que após 30 dias, os animais 02 e 03 foram eutanasiados devido ao prognóstico ruim. Na necropsia dos bovinos 02 e 03 observou-se que o rúmen e o retículo estavam distendidos, o tamanho do omaso e do abomaso estava reduzido e tinha pouco conteúdo. Além disso, observou-se a adesão do retículo ao diafragma (confirmando a causa da lesão do nervo vagal), e o conteúdo ruminal se mostrou homogêneo com aspecto espumoso, consistência viscosa e cor verde oliva (Reis et al., 2016).

Motta et al. (2017) descrevem relato de indigestão vagal em nelore pintado, com $125 \mathrm{~kg}$ e 8 meses de idade criado em sistema extensivo do cerrado no estado do Goiás. Tal animal foi atendido pelo Serviço de Clínica Médica de Grandes Animais da Universidade de Rio Verde- UniRV, com queixa de apatia, inapetência, abdômen distendido, desenvolvimento irregular com a idade, dificuldade respiratória e timpanismo intermitente, não responsivo a sondagem oral. Ao realizar o exame físico foram identificadas: distensão abdominal com aspecto maçã-pera, hipermotilidade ruminal, desidratação severa, ascite acentuada, aceleração dos batimentos cardíacos e da respiração, abafamento de bulhas cardíacas e crepitação pulmonar grossa com linfoadenomegalia generalizada, mucosas hipocoradas e fezes escassas, de coloração verde oliva e com grande quantidade de muco, pele e pelos de baixa qualidade, sem brilhos e vitalidade.

Em decorrência das condições clínicas que o animal apresentava optou-se por intervenção cirúrgica através de laparotomia exploratória pelo flanco esquerdo, no qual foram notadas: peritonite, distensão dos pré-estômagos e abomaso com serosa desvitalizada, com coloração escurecida, presença de conteúdo fibrinoso em toda cavidade, com volume de 12 litros, linfonodos mesentéricos aumentados, com consistência dura e aspecto liso e brilhante. Dessa forma, em função da gravidade do quadro clínico apresentado pelo paciente, optou-se pela eutanásia (Motta et al., 2017).

Como exames complementares solicitou-se hemograma e perfil bioquímico que indicaram leucocitose com neutrofilia, linfocitose, hiperproteinemia com grande aumento nos valores do fibrinogênio e azotemia. Durante a necropsia, evidenciou-se múltiplas áreas de aderências da pleura a cavidade torácica, linfonodos mediastínicos, mesentéricos, pré-hepáticos e renais aumentados, consistentes, lisos e brilhantes com áreas focais de cor vermelho escuro ao corte e pneumonia em hepatização cinzenta. O rúmen estava gravemente distendido, em decorrência do acúmulo de ingesta, com presença de material espumoso e coloração verde amarronzado. Demonstrou-se também grandes áreas com coloração vermelho enegrecida e friável na serosa, com áreas de hemorragia e edema da parede ruminal (Motta et al., 2017).

Coutinho et al. (2019) ao avaliarem diferentes desordens digestivas de natureza mecânica em fêmeas, analisaram 34 vacas mestiças de aptidão leiteira criadas em regime semi-intensivo, em período puerperal, registrando seis animais com quadro de indigestão vagal. Dentre as desordens digestivas estudadas foram constatadas compactação de abomaso/omaso, compactação de rúmen e obstrução intestinal. Valores de colesterol e triglicérides não apresentaram alterações para a espécie bovina para as referidas enfermidades. Porém, condição de hipocalcemia e hiperfosfatemia foi detectada nos animais diagnosticados com indigestão vagal. Durante as análises das 34 vacas apresentaram no exame da atividade sérica das enzimas glutamato desidrogenase (GLDH) e gama glutamiltransferase (GGT) presença de dano hepático. Ademais, denotando a presença de danos teciduais, os animais com indigestão vagal tiveram valores elevados da atividade sérica da Creatino quinase e L-Lactato (outinho et al., 2019). 


\section{Considerações finais}

Nos ruminantes, as doenças do trato gastrointestinal (TGI) constituem um grande problema clínico observado principalmente em sistema semi-intensivo. Em alguns casos, a escolha entre um tratamento clínico, cirúrgico ou ambos é um desafio ímpar. Assim, o uso e conhecimento dos recursos disponíveis são indispensáveis na clínica de ruminantes. Vale ressaltar que o histórico, anamnese e exames complementares contribuem para o diagnóstico. No tratamento, técnicas invasivas podem ser necessárias, contudo, na abordagem terapêutica é necessário considerar a etiologia multifatorial da enfermidade.

\section{Referências bibliográficas}

Berchielli, T. T., Pires, A. V, Oliveira, S. G., \& FUNEP. (2011). Nutrição de Ruminantes (Issue 2th ed.). FUNEP.

Burns, L. V, Helayel, M. A., Silva, M. A. G., Maruo, V. M., Córdova, F. M., Silva, S. L., Barros, C. S. L., \& Ramos, A. T. (2013). Doenças de animais de produção na região centro-norte do Estado de Tocantins: 85 casos. Arquivos de Pesquisa Animall, 2(1), 1-6.

Clauss, M., \& Rössner, G. E. (2014). Old world ruminant morphophysiology, life history, and fossil record: exploring key innovations of a diversification sequence. Annales Zoologici Fennici, 51(1-2), 80-94. https://doi.org/10.5735/086.051.0210.

Constable, P. D., Hinchcliff, K. W., Done, S. H., \& Grünberg, W. (2016). Veterinary medicine-e-book: a textbook of the diseases of cattle, horses, sheep, pigs and goats. Elsevier Health Sciences.

Coutinho, T. L., Mendonça, C. L., Soares, G. S. L., Oliveira Filho, E. F., Souto, R. J. C., Cajueiro, J. F. P., Souza, M. I. S., Silva, N. A. A., Costa, N. A., Soares, P. C., \& Afonso, J. A. B. (2019). Avaliação da bioquímica sanguínea de vacas leiteiras acometidas por desordens digestivas de natureza mecânica. Revista Agrária Acadêmica, 2, 87-100. https://doi.org/10.32406/v2n52019/87100/agrariacad.

Dirksen, G., Grunder, H.-D., \& Stober, M. (2005). Medicina interna y cirugia del bovino. Inter-médica. Dyce, K. M., Wensing, C. J. G., \& Sack, W. O. (2004). Tratado de anatomia veterinária. Elsevier Brasil. Feitosa, F. L. F. (2014). Semiologia veterinária: A arte do diagnóstico. Grupo Gen-Editora Roca Ltda.

Foster, D. (2017). Disorders of rumen distension and dysmotility. Veterinary Clinics of North America: Food Animal Practice, 33, 499-512. https://doi.org/10.1016/j.cvfa.2017.06.006.

Frandson, R. D., Wilke, W. L., \& Fails, A. D. (2011). Anatomia e fisiologia dos animais de fazenda. Guanabara Koogan.

Hedlund, C. H., \& Fossum, T. W. (2008). Cirurgia do sistema digestório. In T. W. Fossum (Ed.), Cirurgia de pequenos animais (pp. 619-672). Elsevier.

Hoflund, S. (1940). Untersuchungen über störungen in den funktionen der er wiederkäuermagen, durch schädigungen des n. vagus verursacht. Svensk. Vet. Tidskr, 45, 322-326.

Hussain, S. A., Uppal, S. K., Sood, N. K., \& Mahajan, S. K. (2014). Clinico hemato biochemical findings, clinical management, and production performance of bovines with late pregnancy indigestion (type IV vagal indigestion). Veterinary Medicine International, 2014, 1-6. https://doi.org/10.1155/2014/525607.

Köning, H. E., \& Liebich, H. G. (2011). Anatomia dos animais domésticos texto e atlas colorido. Editora Artmed.

Motta, R. G., Martins, L. S. A., Gonçalves, I. J., Ribeiro, M. G., Santos, P. A., \& Nardi Júnior, G. (2017). Indigestão vagal em bovino secundária a leucose enzoótica juvenil-relato de caso. VI JornacitecJornada Científica e Tecnológica.

Muzzi, L. A. L., Muzzi, R. A. L., \& Gabellini, E. L. A. (2009). Técnica de fistulação e canulação do rúmen em bovinos e ovinos. Ciência Agrotécnica, 33, 2059-2064.

Perkins, G. A. (2017). Disorders causing abdominal distension in cattle - Vagus indigestion. In S. L. Fubini \& N. G. Ducharme (Eds.), Farm Animal Surgery. Elselvier Saunders.

Radostits, O. M., Gay, C. C., Blood, D. C., Hinchcliff, K. W., \& McKenzie, R. A. (2010). Clínica 
Veterinária: um tratado de doenças dos bovinos, ovinos, suínos, caprinos e eqüinos (Vol. 1). Guanabara Koogan.

Reece, W. O. (2008). Anatomia funcional e fisiologia dos animais domésticos. Editora Roca.

Reece, W. O., \& Penteado Júnior, N. (1996). Fisiologia de animais domésticos. Roca.

Rehage, J., Stockhofe-Zurwieden, N., Kaske, M., \& Yalzin, E. (1992). Hoflund's syndrome. American Association of Bovine Practitioners Proceedings of the Annual Conference, 131-136.

Reis, A. S., Bomjardim, H. dos A., Oliveira, C. M., Oliveira, C. H., Silveira, J. A., Silva, N. D., Salvarani, F. M., Silva, J. B., \& Barbosa, J. D. (2016). Vagal indigestion in Zebu cattle in Brazil. Revista Salud Animal, 38(3), 149-153.

Romão, F. T. N. M. A., Barberini, D. J., \& Gomes, R. G. (2012). Estenose funcional pilórica em vaca leiteira: relato de caso. Revista Acadêmica Ciência Animal, 10(1), 99-103. https://doi.org/10.7213/academica.7535.

Simões, S. V. D., Lira, M. Á. A., Miranda Neto, E. G., Pessoa, C. R. M., Medeiros, G. X., \& Medeiros, J. M. A. (2014). Transtorno motor sugestivo de indigestão vagal em caprino-Relato de caso. Brazilian Journal of Veterinary Medicine, 36(1), 101-104.

Smith, D. F., Bech, J. L., \& Whitlock, R. H. (1992). Anorexia (Hypofagia) with abdominal distention Vagal indigestion and frothy bloat. In N. V. Anderson (Ed.), Veterinary Gastroenterology. Lea \& Febiger.

Smith, M. O. (2006). Tratado de medicina interna de grandes animais (Vol. 1). Manole.

Soares, G. S. L., Costa, N. A., Afonso, J. A. B., Souza, M. I., Cajueiro, J. F. P., Silva, J. C. R., Ferreira, F., \& Mendonça, C. L. (2021). Enfermidades do sistema digestório de bovinos diagnosticadas na Clínica de Bovinos de Garanhuns-UFRPE: estudo retrospectivo e influência da sazonalidade. Pesquisa Veterinária Brasileira, 41. https://doi.org/10.1590/1678-5150-PVB-6800.

Teixeira, J. C. (1996). Fisiologia digestiva dos animais ruminantes. Lavras: UFLA/FAEPE.

Welkoborsky, H. J. (2011). Current aspects in ultrasonography of the salivary glands. HNO, 59(2), 155165. https://doi.org/10.1007/s00106-010-2217-4.

Willard, M. D. (2010). Distúrbios do sistema digestório. In W. Richard, C. Nelson, \& G. Couto (Eds.), Medicina Interna de Pequenos Animais. Elsevier.

Recebido: 7 de agosto de 2021 Aprovado: 17 de setembro de 2021

Disponível online: 9 de novembro de 2021
Licenciamento: Este artigo é publicado na modalidade Acesso Aberto sob a licença Creative Commons Atribuição 4.0 (CC-BY 4.0), a qual permite uso irrestrito, distribuição, reprodução em qualquer meio, desde que o autor e a fonte sejam devidamente creditados. 